\title{
Transition Metal (II) Complexes of $(E)-N-(4-$ methylbenzylidene)-2-((Z)-(4- methylbenzylidene)amino)benzamides: Synthesis, Characterization and their Biological Evaluation
}

\author{
Venkatesh K Bhovi ${ }^{1, * \mathbb{D}}$, Bharathi $\mathrm{K}^{1 \mathbb{D}}$, Sulochana P Melinmath ${ }^{1} \mathbb{D}$, Vrushabendra Basavanna ${ }^{2}$, \\ Srikantamurthy Ningaiah ${ }^{2, *}$ \\ 1 P G Studies and research in Chemistry, Vijayanagar College Hosapete, Vijayanagar, Karnataka, India -583201 \\ 2 Department of Chemistry, Vidyavardhaka College of Engineering, Visvesvaraya Technological University, Mysuru-570 \\ 002, Karnataka, India \\ * Correspondence:venki.kb123@gmail.com (V.K.B.);srijmn@vvce.ac.in(S.N.) \\ Scopus Author ID35096095900;55786395000
}

Received: 31.05.2021; Revised: 5.07.2021; Accepted: 9.07.2021; Published: 10.08.2021

\begin{abstract}
A novel series of transition metal (II) complexes (5a-h) were conveniently synthesized via reaction of important transition metals $(\mathrm{Co}, \mathrm{Cu}, \mathrm{Zn}, \mathrm{Ni})$ with (E)-N-(4-methylbenzylidene)-2-((Z)-(4methylbenzylidene)amino)benzamide Schiff base (3) which was previously synthesized by reacting 2aminobenzohydrazide (1) with 4-methylbenzaldehyde (2). The synthesized metal complexes' structure was elucidated by IR, NMR, mass, and elemental analysis. Additionally, we also evaluated the antioxidant, antimicrobial and antifungal activity of the synthesized metal complexes. The bioassay of the novel transition metal complexes envisioned that compounds5eand5c showed better antimicrobial activity than the free ligand, and compounds5g and 5a showed good activity against most bacterial strains. On the other hand, hydrated metal complexes $5 \mathrm{~b}, 5 \mathrm{~d}, 5 \mathrm{f}$, and $5 \mathrm{~h}$ showed moderate to good antimicrobial activity. In comparison with ascorbic acid, most of the metal complexes showed moderate to good antioxidant activity. The current bioassay was investigated and proved that the compounds $5 \mathrm{e}$ and $5 \mathrm{c}$ as antimicrobial agents act on highly resistant strains of microbes.
\end{abstract}

Keywords: metal (II) complex; Schiff's base; antimicrobial; ascorbic acid.

(C) 2021 by the authors. This article is an open-access article distributed under the terms and conditions of the Creative Commons Attribution (CC BY) license (https://creativecommons.org/licenses/by/4.0/).

\section{Introduction}

Schiff's base is a sub-class of organic imines considered secondary aldimines or ketimines, depending upon the structure [1]. A new era has started in coordination chemistry since 1869 after Schiff's elegant synthesis of azomethane complexes of copper(II) from preformed metal, salicylaldehyde, and primary amine [2]. Schiff base is formed as a condensation product of primary amine with carbonyl compounds [3]. This was first reported by Schiff [4], which contains the $>\mathrm{C}=\mathrm{N}$ - group, which is also called azomethine or imine. The $>\mathrm{C}=\mathrm{N}$ - group, combined with more such groups or others like phenolic $-\mathrm{OH}$ or amino groups, can effectively form metal complexes. The Schiff-based metal complexes have shown evidence of importance significant in inorganic and organic chemistry due to their biological activity. In recent years several reports are published on the preparation of these compounds and their application [5]. Schiff bases of aliphatic aldehydes are comparatively unstable and readily undergo polymerization, whereas aromatic aldehydes possessing effective conjugation are 
stable. In general, aldehyde compounds react faster than ketones in condensation reactions and readily yield Schiff bases as the reaction center of aldehyde is sterically less hindered than ketone [6]. Furthermore, the extra carbon of ketone compounds donates electron density to the azomethine carbon and thus makes the ketone less electrophilic compared to aldehyde [7].

The reactivity of aldehyde towards electrophilic reactions attracts the chemist to synthesize novel Schiff's base, which acts as a ligand by treating with a suitable amine or acid hydrazide. Moreover, Schiff bases have been reported to exhibit a wide variety of biological actions under the azomethine linkage, which is responsible for various antibacterial [8], antifungal [9], antitumor [10], herbicidal [11], and antioxidant [12-13] activity. These compounds were also synthesized to test their inhibition activity against $\alpha$-glucosidase [14]. Similarly, metal complexes procured from Schiff's base and metal salts and screened for their antifungal [15-16], antimicrobial [17], antitumor [18], antioxidant [19-20], antimalarial [21], cytotoxicity [22] activity. These complexes were also tested for the inhibition of human phosphatases [23], cleavage efficiency against pBR322 DNA [24], and for the treatment of hepatocellular carcinoma [25]. The above study on a wide variety of applications of Schiff's base and its metal complexes in the biological field motivated us to synthesize the transition metal (II) complexes through Schiff's base intermediate.

\section{Materials and Methods}

\subsection{Chemistry.}

The present research work focuses on the synthesis of ligands and their transition metal complexes. Also, the details of various physicochemical techniques employed to characterize parent ligands, corresponding complexes, and the experimental protocols followed for the evaluation of biological activity are summarized in detail.

The 4-methylbenzaldehyde and 2-aminobenzohydrazide were purchased from S.D. fine chemical India. The tartaric acid, methanol, and glacial acetic acid were procured from Aldrich, India. All the purchased chemicals were of analytical grade and used as supplied without further purification. Spectral grade deuterated DMSO-d 6 and $\mathrm{CDCl}_{3}$ were used to record the NMR spectra. HPLC grade methanol and acetonitrile were used to record LCMS spectra of the ligand as well as transition metal complexes. Metal chlorides used to prepare complexes in the present work are $\mathrm{CoCl}_{2} .6 \mathrm{H}_{2} \mathrm{O}, \mathrm{CuCl}_{2} .2 \mathrm{H}_{2} \mathrm{O}$, anhydrous $\mathrm{ZnCl}_{2}$ and $\mathrm{NiCl}_{2} .6 \mathrm{H}_{2} \mathrm{O}$. These metal salts were procured from S.D. fine and Spectrochem Chemicals, India. Solvents were purchased from S.D. fine; most of the time, new bottles were taken for ligand and metal complex synthesis. The drying reagents employed at various stages viz., anhydrous sodium sulfate, anhydrous magnesium sulfate, anhydrous calcium chloride, and mineral acids such as hydrochloric acid, sulphuric acid, nitric acid, and bases like sodium hydroxide pellets and ammonia were of analytical grade obtained from S.D. fine Chemicals, India.

Our aim of the experiment is to synthesis and biological studies of mixed ligand metal complexes in two steps by taking two different ligands such as $(E)-N^{\prime}$-(4-methylbenzyldene) 2-[(E)-(4-methylbenzylidene)amino)benzolhydrazide)] (3) and tartaric acid (4). Literature reveals that nitrogen-containing heterocyclic compounds showed good biological activities. The reaction involves two steps, step 1; the synthesis of Schiff base by taking 4methylbenzaldehyde and 2-amino-benzohydrazide in the ratio of 2:1, and the second step involves the preparation of metal complexes by taking Schiff base, tartaric acid the metal chloride in the ratio of 2:1:2 equivalent respectively as shown in the Scheme 1. 


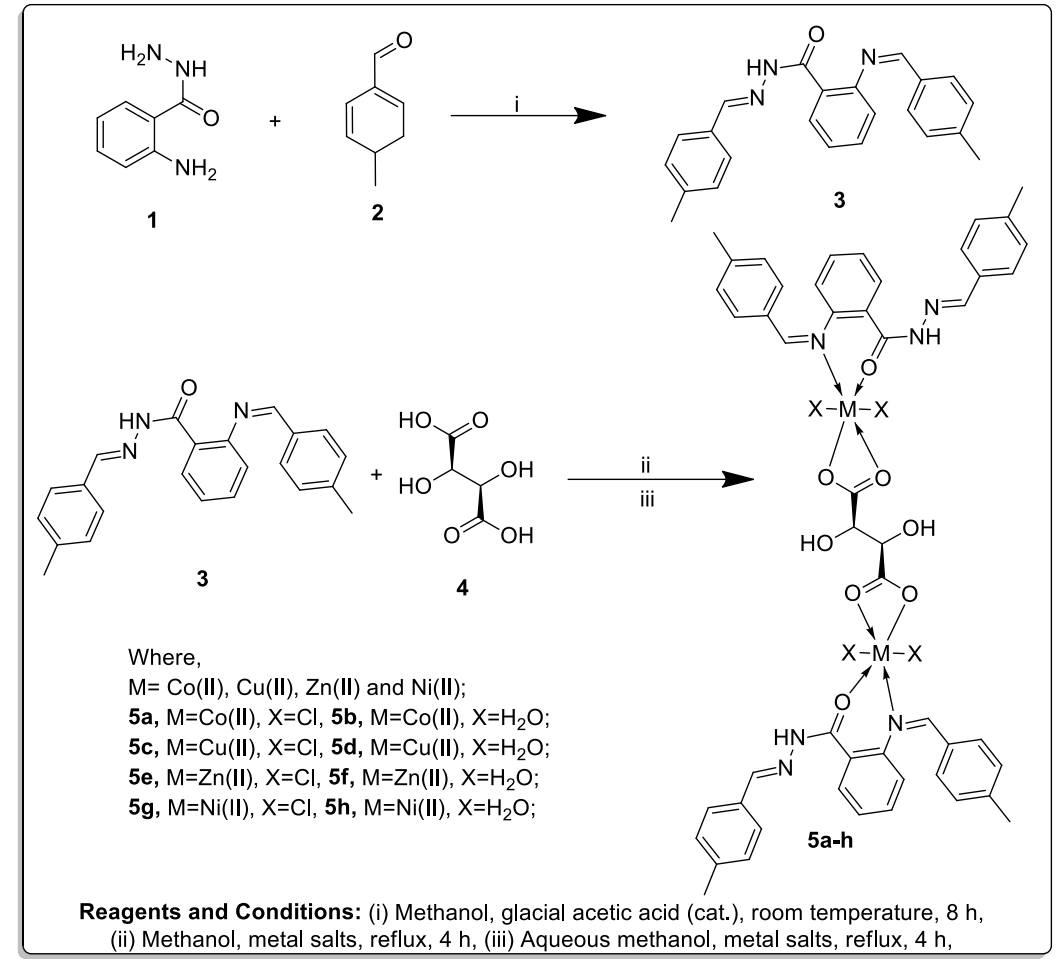

Scheme 1. Synthesis of novel transition metal (II) complexes (5a-h).

2.1.1. Typical procedure for synthesis (E)-N-(4-methylbenzylidene)-2-((Z)-(4methylbenzylidene)amino)benzamide Schiff's base(3).

The methanolic mixture (20 $\mathrm{ml})$ of 2- aminobenzohydrazide $(1,1.51 \mathrm{~g}, 10.00 \mathrm{mmol})$ and 4-methylbenzaldehyde(2,2.40 g, 20.00mmol)was taken in a $50 \mathrm{ml}$ round bottom flask fitted with a water cooled condenser and stirred for 2-3 hours. During the course of the reaction 2-3 drops of acetic acid (catalytic amount)was added. After completion, the solid separated were filtered, washed repeatedly with cold ethanol and dried in air. The purity of the compound (3)was checked by TLC on pre-coated silica gel plate.The crude compound was recrystallized from methanol to procure $(E)-N$-(4-methylbenzylidene)-2-((Z)-(4-methylbenzylidene) amino) benzamide Schiff base (3.07 g) as white solid in $90.2 \%$ yield. m.p: $289^{\circ} \mathrm{C}$. IR (Nujol): 3737 $\mathrm{cm}^{-1}(\mathrm{~N}-\mathrm{H}), 3271 \mathrm{~cm}^{-1}(\mathrm{C}-\mathrm{H}), 3270 \mathrm{~cm}^{-1}(\mathrm{O}-\mathrm{H}), 1656 \mathrm{~cm}^{-1}(\mathrm{C}=\mathrm{O}), 1610 \mathrm{~cm}^{-1}(\mathrm{C}=\mathrm{N}), 1144 \mathrm{~cm}^{-}$ ${ }^{1}$ (C-O); ${ }^{1} \mathrm{H}$ NMR (400 MHz, $\left.\mathrm{CDCl}_{3}\right): \delta 2.34$ (s, 6H, $-\mathrm{CH}_{3}$ ), 7.28-7.29 (d, 4H, ArH), 7.64-7.91 $(\mathrm{m}, 8 \mathrm{H}, \mathrm{ArH}), 8.03(\mathrm{~s}, 1 \mathrm{H}, \mathrm{NH}), 8.18(\mathrm{~s}, 1 \mathrm{H},=\mathrm{CH}-), 8.79$ (s, $1 \mathrm{H},=\mathrm{CH}-) ;{ }^{13} \mathrm{C} \mathrm{NMR}(400 \mathrm{MHz}$, $\left.\mathrm{CDCl}_{3}\right): \delta 20.8,126.4,128.7,129.2,129.9,130.6,131.6,133.1,134.6,141.6,154.1,159.9$, 164.2, 175.8; MS (relative abundance) $\mathrm{m} / \mathrm{z}=356.10[\mathrm{M}+\mathrm{H}]^{+}$; Anal. \% Calc for $\mathrm{C}_{23} \mathrm{H}_{21} \mathrm{~N}_{3} \mathrm{O}: \mathrm{C}$ 77.72, H 5.96, N 11.82, O 4.50; Found: C 77.69, H 5.99, N 11.80, O 4.52.

\subsubsection{Typical procedure for the synthesis of transition metal complexes (5a-h).}

The ligand (E)- $N$-(4-methylbenzylidene)-2-((Z)-(4-methylbenzylidene) amino) benzamide $(3,0.356 \mathrm{~g} 1.00 \mathrm{mmol})$ and tartaric acid $(0.075 \mathrm{~g}, 0.50 \mathrm{mmol})$ were stirred initially with transition metal(II) chlorides in 2:1:1 molar ratio in $20 \mathrm{ml}$ of dry methanol/aqueous methanol solution, and then the reaction mixtures were refluxed on a water bath at $80{ }^{\circ} \mathrm{C}$ for over 3 hours. The color of the reaction mixtures was discharged during the reaction completion. After completion of the reaction, the resultant complexes were filtered, washed with methanol, and then with chloroform and air-dried to afford series of transition metal complexes (5a-h). 


\section{Cobalt metal complex (5a)}

Obtainedfrom(E)-N-(4-methylbenzylidene)-2-((Z)-(4-

methylbenzylidene)amino)benzamide $\quad(3,0.356 \mathrm{~g} 1.00 \mathrm{mmol})$, tartaric acid(0.075 $\mathrm{g}, 0.50$ $\mathrm{mmol})$, cobalt (II) chloride hexahydrate $(0.237 \mathrm{~g}, 1.00 \mathrm{mmol})$ in dry methanol $(20 \mathrm{ml})$ as light pink coloured solid $(0.79 \mathrm{~g})$ in $70.7 \%$ yield. IR (Nujol): $3742 \mathrm{~cm}^{-1}(\mathrm{~N}-\mathrm{H}), 3270 \mathrm{~cm}^{-1}(\mathrm{O}-\mathrm{H})$, $3269 \mathrm{~cm}^{-1}(\mathrm{C}-\mathrm{H}), 1650 \mathrm{~cm}^{-1}(\mathrm{C}=\mathrm{O}), 1613 \mathrm{~cm}^{-1}(\mathrm{C}=\mathrm{N}), 1132 \mathrm{~cm}^{-1}$ (C-O); MS (relative abundance) $\mathrm{m} / \mathrm{z}=1119.0[\mathrm{M}+\mathrm{H}]^{+}$; Anal. \% Calc for $\mathrm{C}_{50} \mathrm{H}_{46} \mathrm{Cl}_{4} \mathrm{Co}_{2} \mathrm{~N}_{6} \mathrm{O}_{8}: \mathrm{C} 53.69, \mathrm{H} \mathrm{4.14, \textrm {Cl }}$ 12.68, Co 10.54, N 7.51, O 11.44; Found: C 53.63, H 4.19, Cl 12.68, Co 10.52, N 7.51, O 11.47 .

\section{Cobalt metal complex (5b)}

Obtained from(E)-N-(4-methylbenzylidene)-2-((Z)-(4-methylbenzylidene) amino) benzamide $(3,0.356 \mathrm{~g} 1.00 \mathrm{mmol})$, tartaric acid(0.075 g,0.50 mmol), cobalt (II) chloride hexahydrate $(0.237 \mathrm{~g}, 1.00 \mathrm{mmol})$ in aqueous methanol $(20 \mathrm{ml})$ as dark pink coloured solid $(0.69 \mathrm{~g})$ in $69.6 \%$ yield. IR (Nujol): $3737 \mathrm{~cm}^{-1}(\mathrm{~N}-\mathrm{H}), 3276 \mathrm{~cm}^{-1}(\mathrm{O}-\mathrm{H}), 3265 \mathrm{~cm}^{-1}(\mathrm{C}-\mathrm{H}), 1661$ $\mathrm{cm}^{-1}(\mathrm{C}=\mathrm{O}), 1621 \mathrm{~cm}^{-1}(\mathrm{C}=\mathrm{N}), 1145 \mathrm{~cm}^{-1}(\mathrm{C}-\mathrm{O}) ; \mathrm{MS}$ (relative abundance) $\mathrm{m} / \mathrm{z}=1050.0$ $[\mathrm{M}+\mathrm{H}]^{+}$; Anal. \% Calc for $\mathrm{C}_{50} \mathrm{H}_{54} \mathrm{Co}_{2} \mathrm{~N}_{6} \mathrm{O}_{12}$ : C 57.26, H 5.19, Co 11.24, N 8.01, O 18.30; Found: C 57.20, H 5.20, Co 11.27, N 8.00, O 18.33.

\section{Copper metal complex (5c)}

Obtained from(E)-N-(4-methylbenzylidene)-2-((Z)-(4-methylbenzylidene) amino) benzamide $(3,0.356 \mathrm{~g} 1.00 \mathrm{mmol})$, tartaric acid(0.075 g,0.50 mmol), copper (II) chloride dihydrate $(0.170 \mathrm{~g}, 1.00 \mathrm{mmol})$ in dry methanol $(20 \mathrm{ml})$ as light red coloured solid $(0.71 \mathrm{~g})$ in $71.9 \%$ yield. IR (Nujol): $3749 \mathrm{~cm}^{-1}(\mathrm{~N}-\mathrm{H}), 3276 \mathrm{~cm}^{-1}(\mathrm{O}-\mathrm{H}), 3263 \mathrm{~cm}^{-1}(\mathrm{C}-\mathrm{H}), 1659 \mathrm{~cm}^{-1}$ $(\mathrm{C}=\mathrm{O}), 1618 \mathrm{~cm}^{-1}(\mathrm{C}=\mathrm{N}), 1127 \mathrm{~cm}^{-1}(\mathrm{C}-\mathrm{O})$; MS (relative abundance) $\mathrm{m} / \mathrm{z}=1128.0[\mathrm{M}+\mathrm{H}]^{+}$; Anal. \% Calc for $\mathrm{C}_{50} \mathrm{H}_{46} \mathrm{Cl}_{4} \mathrm{Cu}_{2} \mathrm{~N}_{6} \mathrm{O}_{8}$ : C 53.25, $\mathrm{H}$ 4.11, $\mathrm{Cl}$ 12.57, $\mathrm{Cu}$ 11.27, N 7.45, O 11.35; Found: C 53.15, H 4.19, Cl 12.53, Cu 11.30, N 7.46, O 11.36.

\section{Copper metal complex $(5 d)$}

Obtained from(E)-N-(4-methylbenzylidene)-2-((Z)-(4-methylbenzylidene) amino) benzamide $(3,0.356 \mathrm{~g} 1.00 \mathrm{mmol})$, tartaric acid $(0.075 \mathrm{~g}, 0.50 \mathrm{mmol})$, copper (II) chloride dehydrate $(0.170 \mathrm{~g}, 1.00 \mathrm{mmol})$ in aqueous methanol $(20 \mathrm{ml})$ as deep red coloured solid $(0.83$ g) in $78.4 \%$ yield. IR (Nujol): $3743 \mathrm{~cm}^{-1}(\mathrm{~N}-\mathrm{H}), 3278 \mathrm{~cm}^{-1}(\mathrm{O}-\mathrm{H}), 3262 \mathrm{~cm}^{-1}(\mathrm{C}-\mathrm{H}), 1658 \mathrm{~cm}^{-}$ ${ }^{1}(\mathrm{C}=\mathrm{O}), 1625 \mathrm{~cm}^{-1}(\mathrm{C}=\mathrm{N}), 1153 \mathrm{~cm}^{-1}(\mathrm{C}-\mathrm{O})$; MS (relative abundance) $\mathrm{m} / \mathrm{z}=1059.0[\mathrm{M}+\mathrm{H}]^{+}$; Anal. \% Calc for $\mathrm{C}_{50} \mathrm{H}_{54} \mathrm{Cu}_{2} \mathrm{~N}_{6} \mathrm{O}_{12}$ : C 56.76, H 5.14, Cu 12.01, N 7.94, O 18.15; Found: C 56.79, H 5.12, Cu 12.03, N 7.93, O 18.13.

\section{Zinc metal complex (5e)}

Obtained from(E)-N-(4-methylbenzylidene)-2-((Z)-(4-methylbenzylidene) amino) benzamide $(3,0.356 \mathrm{~g} 1.00 \mathrm{mmol})$, tartaric acid $(0.075 \mathrm{~g}, 0.50 \mathrm{mmol})$, anhydrous zinc (II) chloride $(0.136 \mathrm{~g}, 1.00 \mathrm{mmol})$ in dry methanol $(20 \mathrm{ml})$ as light green coloured solid $(0.87 \mathrm{~g})$ in $76.9 \%$ yield. IR (Nujol): $3757 \mathrm{~cm}^{-1}(\mathrm{~N}-\mathrm{H}), 3281 \mathrm{~cm}^{-1}(\mathrm{O}-\mathrm{H}), 3263 \mathrm{~cm}^{-1}(\mathrm{C}-\mathrm{H}), 1662 \mathrm{~cm}^{-1}$ $(\mathrm{C}=\mathrm{O}), 1621 \mathrm{~cm}^{-1}(\mathrm{C}=\mathrm{N}), 1135 \mathrm{~cm}^{-1}(\mathrm{C}-\mathrm{O})$; MS (relative abundance) $\mathrm{m} / \mathrm{z}=1132.0[\mathrm{M}+\mathrm{H}]^{+}$; 
Anal. \% Calc for $\mathrm{C}_{50} \mathrm{H}_{46} \mathrm{Cl}_{4} \mathrm{Zn}_{2} \mathrm{~N}_{6} \mathrm{O}_{8}$ : C 53.07, $\mathrm{H}$ 4.10, Cl 12.53, N 7.43, O 11.31, Zn 11.56; Found: C 53.11, H 4.13, Cl 12.50, N 7.44, O 11.31, Zn 11.53.

\section{Zinc metal complex (5f)}

Obtained from(E)-N-(4-methylbenzylidene)-2-((Z)-(4-methylbenzylidene) amino) benzamide $(3,0.356 \mathrm{~g} 1.00 \mathrm{mmol})$, tartaric acid $(0.075 \mathrm{~g}, 0.50 \mathrm{mmol})$, anhydrous zinc (II) chloride $(0.136 \mathrm{~g}, 1.00 \mathrm{mmol})$ in aqueous methanol $(20 \mathrm{ml})$ as light green coloured solid $(0.81$ g) in $76.3 \%$ yield. IR (Nujol): $3747 \mathrm{~cm}^{-1}(\mathrm{~N}-\mathrm{H}), 3289 \mathrm{~cm}^{-1}(\mathrm{O}-\mathrm{H}), 3258 \mathrm{~cm}^{-1}(\mathrm{C}-\mathrm{H}), 1668 \mathrm{~cm}^{-}$ ${ }^{1}(\mathrm{C}=\mathrm{O}), 1619 \mathrm{~cm}^{-1}(\mathrm{C}=\mathrm{N}), 1160 \mathrm{~cm}^{-1}(\mathrm{C}-\mathrm{O})$; MS (relative abundance) $\mathrm{m} / \mathrm{z}=1062.0[\mathrm{M}+\mathrm{H}]^{+}$; Anal. \% Calc for $\mathrm{C}_{50} \mathrm{H}_{54} \mathrm{Zn}_{2} \mathrm{~N}_{6} \mathrm{O}_{12}$ : C 56.56, H 5.13, N 7.92, O 18.08, Zn 12.32; Found: C 56.49, H 5.15, N 7.93, O 18.10, Zn 12.33.

\section{Nickel metal complex $(5 g)$}

Obtained from(E)-N-(4-methylbenzylidene)-2-((Z)-(4-methylbenzylidene) amino) benzamide (3, $0.356 \mathrm{~g} 1.00 \mathrm{mmol})$, tartaric acid(0.075 g,0.50 mmol), nickel (II) chloride hexahydrate $(0.237 \mathrm{~g}, 1.00 \mathrm{mmol})$ in dry methanol $(20 \mathrm{ml})$ as light purple coloured solid $(0.79$ g) in $70.6 \%$ yield. IR (Nujol): $3737 \mathrm{~cm}^{-1}(\mathrm{~N}-\mathrm{H}), 3285 \mathrm{~cm}^{-1}(\mathrm{O}-\mathrm{H}), 3257 \mathrm{~cm}^{-1}(\mathrm{C}-\mathrm{H}), 1658 \mathrm{~cm}^{-}$ ${ }^{1}(\mathrm{C}=\mathrm{O}), 1621 \mathrm{~cm}^{-1}(\mathrm{C}=\mathrm{N}), 1126 \mathrm{~cm}^{-1}(\mathrm{C}-\mathrm{O})$; MS (relative abundance) $\mathrm{m} / \mathrm{z}=1119.0[\mathrm{M}+\mathrm{H}]^{+}$; Anal. \% Calc for $\mathrm{C}_{50} \mathrm{H}_{46} \mathrm{Cl}_{4} \mathrm{~N}_{6} \mathrm{Ni}_{2} \mathrm{O}_{8}$ : C 53.71. H 4.15, Cl 12.68, N 7.52, Ni 10.50, O 11.45; Found: C 53.68. H 4.17, Cl 12.71, N 7.50, Ni 10.47, O 11.48.

\section{Nickel metal complex (5h)}

Obtained from(E)-N-(4-methylbenzylidene)-2-((Z)-(4-methylbenzylidene) amino) benzamide $(3,0.356 \mathrm{~g} 1.00 \mathrm{mmol})$, tartaric acid(0.075 g,0.50 mmol), nickel (II) chloride hexahydrate $(0.237 \mathrm{~g}, 1.00 \mathrm{mmol})$ in aqueous methanol $(20 \mathrm{ml})$ as dark purple coloured solid (0.76 g) in 72.4\% yield. IR (Nujol): $3744 \mathrm{~cm}^{-1}(\mathrm{~N}-\mathrm{H}), 3281 \mathrm{~cm}^{-1}(\mathrm{O}-\mathrm{H}), 3249 \mathrm{~cm}^{-1}(\mathrm{C}-\mathrm{H}), 1668$ $\mathrm{cm}^{-1}(\mathrm{C}=\mathrm{O}), 1619 \mathrm{~cm}^{-1}(\mathrm{C}=\mathrm{N}), 1149 \mathrm{~cm}^{-1}(\mathrm{C}-\mathrm{O})$; MS (relative abundance) $\mathrm{m} / \mathrm{z}=1049.0$ $[\mathrm{M}+\mathrm{H}]^{+}$; Anal. \% Calc for $\mathrm{C}_{50} \mathrm{H}_{54} \mathrm{~N}_{6} \mathrm{Ni}_{2} \mathrm{O}_{12}$ : C 57.28, H 5.19, N 8.02, Ni 11.20, O 18.31; Found: C 57.32, H 5.17, N 8.07, Ni 11.18, O 18.26.

\subsection{Biology.}

\subsubsection{DPPH free radical-scavenging ability assay.}

The radical scavenging activities of various synthesized ligand (3) and transition metal complexes (5a-h) were determined spectrophotometrically by using the DPPH radical as a reagent $(100 \mu \mathrm{L})$ in ethanol, according to the methods followed by Santosh Kumar et al.,(different concentration W/V) [26]. The decrease in absorbance which is induced by antioxidants due to the reduction capacity of DPPH radicals, was measured at $517 \mathrm{~nm}$. Series of test solutions were prepared (10 to $50 \mu \mathrm{L})$ in DMF, and the mixture was incubated for 30min at the black room temperature, and then absorbance was measured $[100 \mu \mathrm{L}$ of the DPPH solution]. The RadicalScavenging Activity (RSA) of each sample was calculated using the following equation.

$$
\% R S A=\frac{A c-A f}{A c} \times 100
$$


where Ac-is the absorbance of the control and Af-is the absorbance of the test sample. The experiment was done in triplicate.

\subsubsection{Antimicrobial activity.}

All the synthesized transition metal complexes (5a-h) along with the intermediate ligand (3), were tested for their in vitro antibacterial activity against clinical bacterial strains such as Staphylococcus aureus (MTCC 96), Bacillus cereus (MTCC 8372)(gram-positive), Klebsiella pneumonia, Escherichia coli (MTCC 724) (gram-negative) by disc diffusion method [27]. First, a suspension solution of the microorganism $(0.1 \mathrm{ml}$ of 108 cells $/ \mathrm{ml})$ was taken in solid media plates provided with filter paper discs. The synthesized compounds were taken in $\mathrm{DMF}$ at 50 to $100 \mu \mathrm{g} / \mathrm{ml}$ concentration and incubated with above-developed media in an incubator for 24 hours. The inhibition zone was recorded in diameter $(\mathrm{mm})$ using Tetracycline as a standard drug. The antifungal activity was carried out through a microdilution method against 4 clinical strains such as Aspergillus niger (MTCC 281), Aspergillus flavus (MTCC 873), Fusarium moniliform (MTCC 156), and Fusarium oxysporum (MTCC 284) by using Nystatin as a standard drug. The activity was performed using 96-well plates containing a Mueller-Hinton broth medium (MHB) blend and different concentrations of compounds at 28 ${ }^{\circ} \mathrm{C}$. Minimum inhibition concentration (MIC) and minimal bacterial concentration $(\mathrm{MBC}) /$ minimal fungicidal concentration $(\mathrm{MFC})$ on agar plates were determined after incubation over 24 hours. The MIC and MBC/MFC values were taken as the mean of three replicates.

\section{Results and Discussion}

\subsection{Chemistry.}

All the newly synthesized compounds $(\mathbf{3}, \mathbf{5 a}-\mathbf{h})$ were characterized by IR, NMR, and mass spectral analysis. The structures assigned to the synthesized compounds were also supported by elemental analysis. In IR spectra, appearance of stretching frequency peaks at $3737-3757 \mathrm{~cm}^{-1}$ for $(\mathrm{N}-\mathrm{H}), 3249-3269 \mathrm{~cm}^{-1}$ for $(\mathrm{C}-\mathrm{H}), 3270-3289 \mathrm{~cm}^{-1}$ for $(\mathrm{O}-\mathrm{H}), 1650-1668$ $\mathrm{cm}^{-1}$ for $(\mathrm{C}=\mathrm{O})$ and $1126-1160 \mathrm{~cm}^{-1}$ for $(\mathrm{C}-\mathrm{O})$ indicated the formation of metal complexes. The appearance of sharp singlets at $\delta 2.34 \mathrm{ppm}$ for the $-\mathrm{CH}_{3}$ group and $\delta 8.03 \mathrm{ppm}$ for the $-\mathrm{NH}$ group indicated the formation of 5a. Two singlets also supported it at $\delta 8.18 \mathrm{ppm}$ and $\delta 8.78$ ppm for azomethine groups. The appearance of a sharp carbon peak at 20.8 for two methyl groups also supported the formation of complex 5a. On the other hand, the appearance of a very week signal at 2.17 for the $\mathrm{H}_{2} \mathrm{O}$ and $-\mathrm{NH}$ - group, in addition to the above characteristic signals, confirmed the formation of hydrated metal complex $\mathbf{5} \mathbf{b}$.

In mass spectra, all the synthesized metal complexes showed molecular ion peaks $(\mathrm{M}+1)$ concerning molecular weight, which supported the structures of the obtained complexes. Similarly, IR, NMR, mass, and elemental analysis supported all the transition metal complexes.

\subsection{Biology.}

All the newly synthesized ligand (3) and transition metal (II) complexes (5a-h) were screened for their antioxidant activity and the results obtained are depicted inTable 1 . The bioassay of the synthesized compounds envisioned that most of the compounds showed 
excellent antioxidant activity. The maximum antioxidant activity was observed in the order of $\mathbf{5 e}, \mathbf{5 c}, \mathbf{5 g}$, and $\mathbf{5 a}$. This may be due to the holding of X-type ligands such as chloride by metal (II) complexes. Among the tested compounds, transition metal complexes $\mathbf{5 e}$ and $\mathbf{5 c}$ exhibited excellent interaction with stable DPPH radicals. This may be due to the presence of enzymatic $\mathrm{Zn}$ (II) and $\mathrm{Cu}$ (II) metal ions in addition to X-type ligands, such as chloride in the metal complex. Compared to other metal complexes having neutral ligand $\left(\mathrm{H}_{2} \mathrm{O}\right)$ such as $\mathbf{5 b}, \mathbf{5 d}, \mathbf{5 f}$, 5h, metal complex 5g and 5a holding $X$ type ligand (chloride) exhibited moderate activity, comparable with the standard drug.

All other metal complexes $\mathbf{5 b}, \mathbf{5 d}, \mathbf{5 f}, \mathbf{5 h}$, including newly synthesized ligand $\mathbf{3}$, showed poor antioxidant activity compared to standard drug ascorbic acid. This may be due to the presence of neutral water molecules in the metal complex.

In addition, all the newly synthesized ligand (3) and transition metal complexes (5a-h) were tested for their antimicrobial efficacy disc diffusion method. The results of antibacterial and antifungal activity are depicted in Table 2 and Table 3, respectively. The measured minimal inhibitory concentration (MIC), minimal bactericidal concentration (MBC), and minimal fungicidal concentration (MFC) values are represented in Table 4. The antimicrobial assay carried out against microbes was comparable with antioxidant activity. Among all five tested bacterial strains, gram-positive and gram-negative bacteria were inhibited mostly by metal complexes 5e, 5c, 5g, and 5a. The results envisioned that the complexes5e and $\mathbf{5 c}$ showed excellently and $\mathbf{5 g}$ and $\mathbf{5 a}$ showed good antimicrobial activity against all the tested strains of microbes. The activity is mainly affected by enzymatic metals such as $\mathrm{Zn}(\mathrm{II}), \mathrm{Cu}(\mathrm{II})$, and Xtype ligand in the final compounds. Including the newly synthesized ligand (3), all other metal complexes with neutral ligands (water) such as 5b, 5d, 5f, and $5 \mathrm{~h}$ showed moderate antimicrobial activity compared to standard drugs.

Table 1. Antioxidant activity of synthesized ligand (3) and transition metal (II) complexes (5a-h).

\begin{tabular}{|c|c|c|c|c|c|c|c|}
\hline \multirow[b]{2}{*}{ Products } & \multirow[b]{2}{*}{$\mathbf{M}$} & \multirow[b]{2}{*}{$\mathbf{X}$} & \multicolumn{5}{|c|}{ \% DPPH radical scavenging assay } \\
\hline & & & $\begin{array}{l}\mathbf{1 0} \mu \mathrm{g} / \mathrm{mL} \\
\pm \mathrm{SD}\end{array}$ & $\begin{array}{l}20 \mu \mathrm{g} / \mathrm{mL} \\
\pm \mathrm{SD}\end{array}$ & $\begin{array}{l}\mathbf{3 0} \mu \mathrm{g} / \mathrm{mL} \\
\pm \mathrm{SD}\end{array}$ & $\begin{array}{l}\mathbf{4 0} \mu \mathrm{g} / \mathrm{mL} \\
\pm \mathrm{SD}\end{array}$ & $\begin{array}{l}\mathbf{5 0} \mu \mathrm{g} / \mathrm{mL} \\
\pm \mathrm{SD}\end{array}$ \\
\hline 3 & - & - & $19 \pm 0.032$ & $21 \pm 0.012$ & $27 \pm 0.018$ & $31 \pm 0.097$ & $42 \pm 0.037$ \\
\hline $5 \mathbf{a}$ & Co & $\mathrm{Cl}$ & $25 \pm 0.017$ & $28 \pm 0.021$ & $35 \pm 0.002$ & $43 \pm 0.082$ & $46 \pm 0.029$ \\
\hline $5 \mathbf{b}$ & Co & $\mathrm{H}_{2} \mathrm{O}$ & $18 \pm 0.024$ & $19 \pm 0.009$ & $31 \pm 0.027$ & $39 \pm 0.275$ & $44 \pm 0.121$ \\
\hline $5 c$ & $\mathrm{Cu}$ & $\mathrm{Cl}$ & $30 \pm 0.025$ & $33 \pm 0.033$ & $39 \pm 0.068$ & $50 \pm 0.006$ & $55 \pm 0.033$ \\
\hline $5 d$ & $\mathrm{Cu}$ & $\mathrm{H}_{2} \mathrm{O}$ & $22 \pm 0.019$ & $24 \pm 0.025$ & $29 \pm 0.009$ & $40 \pm 0.053$ & $43 \pm 0.013$ \\
\hline $5 e$ & $\mathrm{Zn}$ & $\mathrm{Cl}$ & $33 \pm 0.027$ & $39 \pm 0.017$ & $43 \pm 0.023$ & $54 \pm 0.112$ & $64 \pm 0.039$ \\
\hline $5 f$ & $\mathrm{Zn}$ & $\mathrm{H}_{2} \mathrm{O}$ & $22 \pm 0.115$ & $25 \pm 0.032$ & $33 \pm 0.039$ & $39 \pm 0.087$ & $42 \pm 0.012$ \\
\hline $5 \mathrm{~g}$ & $\mathrm{Ni}$ & $\mathrm{Cl}$ & $27 \pm 0.028$ & $30 \pm 0.008$ & $37 \pm 0.076$ & $46 \pm 0.053$ & $52 \pm 0.023$ \\
\hline $5 \mathbf{h}$ & $\mathrm{Ni}$ & $\mathrm{H}_{2} \mathrm{O}$ & $15 \pm 0.141$ & $18 \pm 0.123$ & $28 \pm 0.032$ & $41 \pm 0.042$ & $40 \pm 0.043$ \\
\hline Ascorbic acid & ---- & & $31 \pm 0.033$ & $36 \pm 0.021$ & $43 \pm 0.026$ & $51 \pm 0.033$ & $58 \pm 0.005$ \\
\hline
\end{tabular}

Table 2. Inhibitory zone (diameter) mm of synthesized ligand (3) and transition metal (II) complexes (5a-h) against tested bacterial strains. 


\begin{tabular}{|c|c|c|c|c|c|c|c|c|c|c|}
\hline \multirow{4}{*}{ Products } & \multirow{4}{*}{ M } & \multirow{4}{*}{$\mathbf{X}$} & \multicolumn{8}{|c|}{ Antibacterial activity } \\
\hline & & & \multicolumn{4}{|c|}{ Gram-positive } & \multicolumn{4}{|c|}{ Gram-negative } \\
\hline & & & \multicolumn{2}{|c|}{ B. cereus } & \multicolumn{2}{|c|}{ S. aureus } & \multicolumn{2}{|c|}{ E. coli } & \multicolumn{2}{|c|}{ K. pneumonia } \\
\hline & & & $\begin{array}{c}\mathbf{5 0} \mu \mathrm{g} / \mathrm{mL} \\
\pm \mathrm{SD}\end{array}$ & $\begin{array}{c}100 \mu \mathrm{g} / \mathrm{mL} \\
\pm \mathrm{SD}\end{array}$ & $\begin{array}{c}\mathbf{5 0} \mu \mathrm{g} / \mathrm{mL} \\
\pm \mathrm{SD} \\
\end{array}$ & $\begin{array}{c}100 \mu \mathrm{g} / \mathrm{mL} \\
\pm \mathrm{SD}\end{array}$ & $\begin{array}{c}\mathbf{5 0} \mu \mathrm{g} / \mathrm{mL} \\
\pm \mathrm{SD} \\
\end{array}$ & $\begin{array}{c}100 \mu \mathrm{g} / \mathrm{mL} \\
\pm \mathrm{SD}\end{array}$ & $\begin{array}{c}\mathbf{5 0} \mu \mathrm{g} / \mathrm{mL} \\
\pm \mathrm{SD}\end{array}$ & $\begin{array}{c}100 \mu \mathrm{g} / \mathrm{mL} \\
\pm \mathrm{SD}\end{array}$ \\
\hline 3 & - & - & $04 \pm 0.23$ & $09 \pm 0.17$ & $06 \pm 0.15$ & $10 \pm 0.18$ & $12 \pm 0.09$ & $15 \pm 0.12$ & $08 \pm 0.12$ & $14 \pm 0.11$ \\
\hline $5 \mathbf{a}$ & $\mathrm{Co}$ & $\mathrm{Cl}$ & $10 \pm 0.25$ & $11 \pm 0.25$ & $14 \pm 0.24$ & $15 \pm 0.24$ & $13 \pm 0.22$ & $09 \pm 0.26$ & $04 \pm 0.08$ & $06 \pm 0.24$ \\
\hline $\mathbf{5 b}$ & $\mathrm{Co}$ & $\mathrm{H}_{2} \mathrm{O}$ & $05 \pm 0.09$ & $06 \pm 0.15$ & $04 \pm 0.14$ & $05 \pm 0.17$ & $07 \pm 0.17$ & $09 \pm 0.08$ & $02 \pm 0.19$ & $04 \pm 0.13$ \\
\hline $5 \mathrm{c}$ & $\mathrm{Cu}$ & $\mathrm{Cl}$ & $14 \pm 0.16$ & $13 \pm 0.11$ & $15 \pm 0.21$ & $19 \pm 0.19$ & $21 \pm 0.07$ & $17 \pm 0.06$ & $15 \pm 0.12$ & $18 \pm 0.15$ \\
\hline $5 d$ & $\mathrm{Cu}$ & $\mathrm{H}_{2} \mathrm{O}$ & $04 \pm 0.12$ & $07 \pm 0.26$ & $05 \pm 0.32$ & $09 \pm 0.22$ & $07 \pm 0.14$ & $07 \pm 0.16$ & $02 \pm 0.10$ & $07 \pm 0.19$ \\
\hline $5 e$ & $\mathrm{Zn}$ & $\mathrm{Cl}$ & $16 \pm 0.07$ & $19 \pm 0.21$ & $17 \pm 0.09$ & $19 \pm 0.14$ & $23 \pm 0.08$ & $29 \pm 0.18$ & $17 \pm 0.19$ & $21 \pm 0.06$ \\
\hline 5f & $\mathrm{Zn}$ & $\mathrm{H}_{2} \mathrm{O}$ & $03 \pm 0.14$ & $05 \pm 0.24$ & $06 \pm 0.25$ & $08 \pm 0.15$ & $07 \pm 0.14$ & $10 \pm 0.09$ & $07 \pm 0.24$ & $09 \pm 0.27$ \\
\hline $5 \mathrm{~g}$ & $\mathrm{Ni}$ & $\mathrm{Cl}$ & $12 \pm 0.17$ & $21 \pm 0.17$ & $09 \pm 0.25$ & $18 \pm 0.12$ & $18 \pm 0.23$ & $19 \pm 0.14$ & $07 \pm 0.32$ & $14 \pm 0.06$ \\
\hline $5 \mathrm{~h}$ & $\mathrm{Ni}$ & $\mathrm{H}_{2} \mathrm{O}$ & $06 \pm 0.09$ & $10 \pm 0.32$ & $08 \pm 0.15$ & $12 \pm 0.19$ & $10 \pm 0.24$ & $13 \pm 0.28$ & $04 \pm 0.16$ & $06 \pm 0.29$ \\
\hline $\begin{array}{c}\text { Tetracyclin } \\
e\end{array}$ & & & $12 \pm 0.19$ & $20 \pm 0.07$ & $10 \pm 0.21$ & $19 \pm 0.28$ & $19 \pm 0.08$ & $28 \pm 0.09$ & $13 \pm 0.22$ & $21 \pm 0.24$ \\
\hline
\end{tabular}

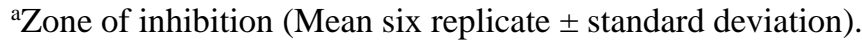

Table 3. Inhibitory zone (diameter) mm of synthesized ligand (3) and transition metal (II) complexes (5a-h) against tested fungal strains.

\begin{tabular}{|c|c|c|c|c|c|c|c|c|}
\hline \multirow{3}{*}{ Products } & \multicolumn{8}{|c|}{ Antifungal activity } \\
\hline & \multicolumn{2}{|c|}{ A. flavus } & \multicolumn{2}{|c|}{ A. niger } & \multicolumn{2}{|c|}{ F oxysporum } & \multicolumn{2}{|c|}{ F monaliforme } \\
\hline & $\begin{array}{c}\mathbf{5 0} \mu \mathrm{g} / \mathrm{mL} \\
\pm \mathrm{SD}\end{array}$ & $\begin{array}{c}100 \mu \mathrm{g} / \mathrm{mL} \\
\pm \mathrm{SD}\end{array}$ & $\begin{array}{c}\mathbf{5 0} \mu \mathrm{g} / \mathrm{mL} \\
\pm \mathrm{SD}\end{array}$ & $\begin{array}{c}100 \mu \mathrm{g} / \mathrm{mL} \\
\pm \mathrm{SD}\end{array}$ & $\begin{array}{c}\mathbf{5 0} \mu \mathrm{g} / \mathrm{mL} \\
\pm \mathrm{SD}\end{array}$ & $\begin{array}{c}100 \mu \mathrm{g} / \mathrm{mL} \\
\pm \mathrm{SD}\end{array}$ & $\begin{array}{c}\mathbf{5 0} \mu \mathrm{g} / \mathrm{mL} \\
\pm \mathrm{SD}\end{array}$ & $\begin{array}{c}\mathbf{1 0 0} \mu \mathrm{g} / \mathrm{mL} \\
\pm \mathrm{SD}\end{array}$ \\
\hline 3 & $03 \pm 0.16$ & $05 \pm 0.12$ & $03 \pm 0.24$ & $05 \pm 0.18$ & $05 \pm 0.14$ & $05 \pm 0.16$ & $06 \pm 0.27$ & $07 \pm 0.26$ \\
\hline $\mathbf{5 a}$ & $05 \pm 0.11$ & $06 \pm 0.21$ & $05 \pm 0.16$ & $06 \pm 0.13$ & $05 \pm 0.18$ & $08 \pm 0.16$ & $06 \pm 0.28$ & $09 \pm 0.09$ \\
\hline $5 \mathbf{b}$ & $10 \pm 0.11$ & $12 \pm 0.21$ & $08 \pm 0.16$ & $14 \pm 0.13$ & $08 \pm 0.18$ & $10 \pm 0.16$ & $06 \pm 0.28$ & $09 \pm 0.09$ \\
\hline $5 c$ & $08 \pm 0.13$ & $09 \pm 0.12$ & $04 \pm 0.13$ & $05 \pm 0.26$ & $05 \pm 0.33$ & $07 \pm 0.22$ & $03 \pm 0.14$ & $06 \pm 0.17$ \\
\hline $5 d$ & $02 \pm 0.19$ & $03 \pm 0.15$ & $03 \pm 0.29$ & $05 \pm 0.13$ & $04 \pm 0.11$ & $03 \pm 0.29$ & $04 \pm 0.13$ & $04 \pm 0.19$ \\
\hline $5 e$ & $11 \pm 0.09$ & $14 \pm 0.26$ & $09 \pm 0.07$ & $15 \pm 0.17$ & $14 \pm 0.11$ & $19 \pm 0.18$ & $08 \pm 0.07$ & $12 \pm 0.22$ \\
\hline 5f & $03 \pm 0.22$ & $05 \pm 0.07$ & $02 \pm 0.23$ & $06 \pm 0.15$ & $02 \pm 0.17$ & $03 \pm 0.38$ & $04 \pm 0.27$ & $06 \pm 0.22$ \\
\hline $5 g$ & $06 \pm 0.22$ & $09 \pm 0.21$ & $07 \pm 0.08$ & $10 \pm 0.12$ & $08 \pm 0.14$ & $11 \pm 0.22$ & $06 \pm 0.25$ & $07 \pm 0.32$ \\
\hline $5 \mathrm{sh}$ & $04 \pm 0.06$ & $05 \pm 0.34$ & $04 \pm 0.11$ & $07 \pm 0.22$ & $03 \pm 0.22$ & $05 \pm 0.23$ & $02 \pm 0.25$ & $03 \pm 0.32$ \\
\hline Nystatin & $15 \pm 0.12$ & $20 \pm 0.23$ & $12 \pm 0.09$ & $27 \pm 0.21$ & $15 \pm 0.18$ & $32 \pm 0.16$ & $11 \pm 0.07$ & $26 \pm 0.14$ \\
\hline
\end{tabular}

Table 4. The minimal inhibitory concentration (MIC), minimal bactericidal concentration (MBC), and minimal fungicidal concentration (MFC) in $\mu \mathrm{g} / \mathrm{mL}$ of synthesized ligand (3) and transition metal (II) complexes (5a-h) against tested strains.

\begin{tabular}{|c|c|c|c|c|c|c|c|c|c|c|c|c|c|c|c|c|}
\hline \multirow{4}{*}{$\begin{array}{c}\text { Compoun } \\
\text { ds }\end{array}$} & \multicolumn{8}{|c|}{ Antibacterial activity } & \multirow{2}{*}{\multicolumn{8}{|c|}{ Antifungal activity }} \\
\hline & \multicolumn{4}{|c|}{ Gram-positive } & \multicolumn{4}{|c|}{ Gram-negative } & & & & & & & & \\
\hline & \multicolumn{2}{|c|}{ B. cereus } & \multicolumn{2}{|c|}{ S. aureus } & \multicolumn{2}{|c|}{ E. coli } & \multicolumn{2}{|c|}{$\begin{array}{c}K . \\
\text { pneumonia }\end{array}$} & \multicolumn{2}{|c|}{ A. flavus } & \multicolumn{2}{|c|}{ A. niger } & \multicolumn{2}{|c|}{$\begin{array}{c}F . \\
\text { oxysporum }\end{array}$} & \multicolumn{2}{|c|}{$\begin{array}{c}F . \\
\text { monaliforme }\end{array}$} \\
\hline & $\begin{array}{c}\text { MI } \\
\text { C }\end{array}$ & MBC & $\begin{array}{c}\text { MI } \\
\text { C }\end{array}$ & MBC & $\begin{array}{c}\text { MI } \\
\text { C }\end{array}$ & MBC & $\begin{array}{c}\text { MI } \\
\text { C }\end{array}$ & MBC & $\underset{\mathrm{C}}{\mathrm{MI}}$ & MFC & $\begin{array}{c}\text { MI } \\
\text { C }\end{array}$ & MFC & $\begin{array}{c}\text { MI } \\
\text { C }\end{array}$ & $\begin{array}{c}\text { MF } \\
\text { C }\end{array}$ & $\begin{array}{c}\text { MI } \\
\text { C }\end{array}$ & MFC \\
\hline 3 & 40 & 255 & 50 & 270 & 35 & 195 & 45 & 240 & 35 & 215 & 50 & 265 & 35 & 195 & 30 & 245 \\
\hline $5 \mathbf{a}$ & 35 & 190 & 35 & 205 & 35 & 175 & 30 & 170 & 45 & 225 & 35 & 230 & 35 & 230 & 30 & 210 \\
\hline $\mathbf{5 b}$ & 50 & 170 & 55 & 255 & 45 & 220 & 40 & 255 & 40 & 245 & 35 & 225 & 35 & 175 & 40 & 240 \\
\hline $5 c$ & 25 & 145 & 30 & 165 & 20 & 125 & 25 & 145 & 30 & 130 & 25 & 190 & 20 & 155 & 25 & 135 \\
\hline 5d & 55 & 240 & 45 & 230 & 50 & 270 & 55 & 260 & 30 & 225 & 55 & 235 & 50 & 250 & 40 & 270 \\
\hline $5 e$ & 20 & 135 & 25 & 135 & 25 & 160 & 20 & 125 & 20 & 125 & 20 & 120 & 20 & 140 & 25 & 120 \\
\hline $5 f$ & 55 & 210 & 40 & 220 & 45 & 250 & 40 & 230 & 45 & 230 & 40 & 210 & 40 & 210 & 35 & 220 \\
\hline $5 \mathrm{~g}$ & 30 & 160 & 30 & 165 & 20 & 205 & 25 & 165 & 20 & 185 & 25 & 150 & 25 & 155 & 20 & 130 \\
\hline $5 \mathrm{~h}$ & 40 & 230 & 35 & 260 & 50 & 220 & 40 & 260 & 40 & 235 & 50 & 245 & 45 & 220 & 45 & 230 \\
\hline
\end{tabular}




\begin{tabular}{|c|c|c|c|c|c|c|c|c|c|c|c|c|c|c|c|c|}
\hline \multirow{4}{*}{$\begin{array}{c}\text { Compoun } \\
\text { ds }\end{array}$} & \multicolumn{8}{|c|}{ Antibacterial activity } & \multirow{2}{*}{\multicolumn{8}{|c|}{ Antifungal activity }} \\
\hline & \multicolumn{4}{|c|}{ Gram-positive } & \multicolumn{4}{|c|}{ Gram-negative } & & & & & & & & \\
\hline & \multicolumn{2}{|c|}{ B. cereus } & \multicolumn{2}{|c|}{ S. aureus } & \multicolumn{2}{|c|}{ E. coli } & \multicolumn{2}{|c|}{$\begin{array}{c}K . \\
\text { pneumonia }\end{array}$} & \multicolumn{2}{|c|}{ A. flavus } & \multicolumn{2}{|c|}{ A. niger } & \multicolumn{2}{|c|}{$\begin{array}{c}F . \\
\text { oxysporum }\end{array}$} & \multicolumn{2}{|c|}{$\begin{array}{c}F . \\
\text { monaliforme }\end{array}$} \\
\hline & $\begin{array}{c}\text { MI } \\
\text { C }\end{array}$ & MBC & $\begin{array}{c}\text { MI } \\
\text { C }\end{array}$ & MBC & $\begin{array}{c}\text { MI } \\
\text { C }\end{array}$ & MBC & $\begin{array}{c}\text { MI } \\
\text { C }\end{array}$ & MBC & $\begin{array}{c}\text { MI } \\
\text { C }\end{array}$ & MFC & $\begin{array}{c}\text { MI } \\
\text { C }\end{array}$ & MFC & $\begin{array}{c}\text { MI } \\
\text { C }\end{array}$ & $\begin{array}{c}\text { MF } \\
\text { C }\end{array}$ & $\begin{array}{c}\text { MI } \\
\text { C }\end{array}$ & MFC \\
\hline $\begin{array}{c}\text { Tetracycli } \\
\text { ne }\end{array}$ & 5 & 135 & 15 & 110 & 15 & 130 & 9 & 110 & $\begin{array}{ll}-- \\
--\end{array}$ & --- & $\begin{array}{ll}-- \\
-1\end{array}$ & --- & $\begin{array}{ll}-- \\
-1\end{array}$ & $\begin{array}{ll}-- \\
\end{array}$ & $\begin{array}{ll}-- \\
\end{array}$ & --- \\
\hline Nystatin & --- & --- & --- & --- & --- & --- & --- & --- & 07 & 120 & 12 & 120 & 20 & 110 & 10 & 110 \\
\hline
\end{tabular}

\section{Conclusions}

We have described the simple synthetic method for the preparation of transition metal (II) complexes by treating series of transition metals such as $\mathrm{Co}(\mathrm{II}), \mathrm{Cu}(\mathrm{II}), \mathrm{Zn}$ (II), Ni(II) with a simple Schiff's base which was initially obtained by the condensation reaction of 4methylbenzaldehyde with 2-aminobenzohydrazide at elevated temperature. The newly synthesized ligand has been screened for antioxidant, antimicrobial, and antifungal activities along with its metal complexes. The bioassay of the synthesized compounds envisioned that 5e and 5c emerged as excellent antimicrobial agents.

\section{Funding}

This research received no external funding.

\section{Acknowledgments}

The authors are thankful for the V V Sangh'S Ballari and Vijayanagar College Hosapete for providing necessary facilities and constant encouragement.

\section{Conflicts of Interest}

The authors declare no conflict of interest.

\section{References}

1. Matsumoto, Y.; Sawamura, J.; Murata, Y.; Nishikata, T.; Yazaki, R.; Ohshima, T. Amino Acid Schiff Base Bearing Benzophenone Imine As a Platform for Highly Congested Unnatural $\alpha$-Amino Acid Synthesis. J. Am. Chem. Soc.2020, 142, 8498-8505, https://doi.org/10.1021/jacs.0c02707.

2. MacLachlan, M.J.; Park, M.K.; Thompson, L.K. Coordination compounds of Schiff-base ligands derived from diaminomaleonitrile (DMN): mononuclear, dinuclear, and macrocyclic derivatives. Inorg. Chem. 1996, 35, 5492-5499, https://doi.org/10.1021/ic960237p.

3. Hamil, A.; Khalifa, K.M.; Almutaleb, A.A.; Nouradean, M.Q. Synthesis, Characterization, and antibacterial activity studies of some transition metal chelates of $\mathrm{Mn}$ (II), $\mathrm{Ni}$ (II), and $\mathrm{Cu}$ (II) with Schiff base derived from diacetylmonoxime with O-phenylenediamine. Adv. J. Chem. A. 2020, 3, 524-533, https://dx.doi.org/10.33945/SAMI/AJCA.2020.4.13.

4. Schiff, H. MittheilungenausdemUniversitätslaboratorium in Pisa: eineneueReiheorganischer Basen. Justus Liebigs Ann. Chem. 1864, 131, 118-119, https://doi.org/10.1002/jlac.18641310113.

5. Shamsipur, M.; Ghiasvand, A.R.; Sharghi, H.; Naeimi. H. Solid-phase extraction of ultra-trace copper (II) using octadecyl silica membrane disks modified by a naphthol-derivative Schiff's base. Anal. Chim. Acta. 2000, 408, 271-277, https://doi.org/10.1016/S0003-2670(99)00873-9.

6. Mahmoudi, F.; Farhadi, S.; Dusek, M.; Poupon, M. Synthesis, Spectroscopy and X-ray Crystallography Structure of Pyridine 4-Carbaldehyde Semicarbazone Schiff Base Ligand. Adv. J. Chem. A.2020, 3, 534-541, https://doi.org/10.33945/SAMI/AJCA.2020.4.14. 
7. Xia, Y.; Wang, J. Transition-metal-catalyzed cross-coupling with ketones or aldehydes via N-tosylhydrazones. J. Am. Chem. Soc.2020, 142, 10592-10605, https://doi.org/10.1021/jacs.0c04445.

8. Hamed, A.A.; Abdelhamid, I.A.; Saad, G.R.; Elkady, N.A.; Elsabee, M.Z. Synthesis, characterization, and antimicrobial activity of a novel chitosan Schiff bases based on heterocyclic moieties. Int. J. Biol. Macromol. 2020, 153, 492-501, https://doi.org/10.1016/j.ijbiomac.2020.02.302.

9. Magalhaes, T.F.F.; da Silva, C.M.; Dos Santos, L.B.; Santos, D.A.; Silva, L.; Fuchs, B.B.; Mylonakis, E.; Martins, C.V.; de Resende-Stoianoff, M.A.; de Fatima, A. Cinnamyl Schiff bases: synthesis, cytotoxic effects and antifungal activity of clinical interest. Lett. Appl. Microbiol. 2020, 71, 490-497, https://doi.org/10.1111/lam.13356.

10. Hassan, A.M.; Osman Said, A.; Heakal, B.H.; Younis, A.; Abdelmoaz, M.A.; Abdrabou, M.M. Conventional and Microwave-Assisted Synthesis, Antimicrobial and Antitumor Studies of Tridentate Schiff Base Derived from O-vanillin and Phenyl Urea and its Complexes. Adv. J. Chem. A. 2020, 3, 621-638, https://doi.org/10.22034/AJCA.2020.105996.

11. Zhu, S.; Xu, S.; Yi, X.; Wang, J.; Zhao, Z.; Jiang, J. High value-added application of turpentine as a potential renewable source for the synthesis of heterocyclic Schiff base derivatives of cis-1, 8-p-menthane-diamine serving as botanical herbicides. Ind. Crops. Prod. 2018, 115, 111-116, https://doi.org/10.1016/j.indcrop.2018.02.021.

12. Beyazit, N.; Çakran, H.S.; Cabir, A.; Akiscan, Y.; Demetgul, C. Synthesis, characterization and antioxidant activity of chitosan Schiff base derivatives bearing (-)-gossypol. Carbohydr. Polym. 2020, 240, 116333, https://doi.org/10.1016/j.carbpol.2020.116333.

13. Yuksel, E.; Bilici, A.; Gecibesler, I.H.; Kaya. I. Synthesis and antioxidant activities of phenolic Schiff base monomers and polymers. Can. J. Chem. 2020, 98, 151-157, https://doi.org/10.1139/cjc-2019-0307.

14. Tokali, F.S.; Taslimi, P.; Usanmaz, H.; Karaman, M.; Sendil, K. Synthesis, characterization, biological activity and molecular docking studies of novel Schiff bases derived from thiosemicarbazide: Biochemical and computational approach. J. Mol. Struct. 2021, 1231, 129666, https://doi.org/10.1016/j.molstruc.2020.129666.

15. Joshi, R.; Kumari, A.; Singh, K.; Mishra, H.; Pokharia, S. Triorganotin (IV) complexes of Schiff base derived from 1, 2, 4-triazole moiety: Synthesis, spectroscopic investigation, DFT studies, antifungal activity, and molecular docking studies. J. Mol. Struct. 2020,1206, https://doi.org/10.1016/j.molstruc.2019.127639.

16. Frei, A.; King, A.P.; Lowe, G.J.; Cain, A.K.; Short, F.L.; Dinh, H.; Elliott, A.G.; Zuegg, J.; Wilson, J.J.; Blaskovich, M.A. Nontoxic Cobalt (III) Schiff Base Complexes with Broad-Spectrum Antifungal Activity. Chem. Eur. J. 2021, 27, 2021-2029, https://dx.doi.org/10.1002\%2Fchem.202003545.

17. Singh, K.; Barwa, M.S.; Tyagi, P. Synthesis and characterization of cobalt (II), nickel (II), copper (II), and zinc (II) complexes with Schiff base derived from 4-amino-3-mercapto-6-methyl-5-oxo-1, 2, 4-triazine. Eur. J. Med. Chem. 2007, 42, 394-402, https://doi.org/10.1016/j.ejmech.2006.10.016.

18. Barbosa, H.F.; Attjioui, M.; Ferreira, A.P.; Moerschbacher, B.M.; Cavalheiro, E.T. New series of metal complexes by amphiphilic biopolymeric Schiff bases from modified chitosans: Preparation, characterization, and effect of molecular weight on its biological applications. Int. J. Biol. Macromol. 2020, 145, 417-428, https://doi.org/10.1016/j.ijbiomac.2019.12.153.

19. Buldurun, K.; Turan, N.; Bursal, E.; Mantarci, A.; Turkan, F.; Taslimi, P.; Gulcin, I. Synthesis, spectroscopic properties, crystal structures, antioxidant activities and enzyme inhibition determination of Co (II) and Fe (II) complexes of Schiff base. Res. Chem. Intermed. 2020, 46, 283-297, https://doi.org/10.1007/s11164-01903949-3.

20. Buldurun, K.; Turan, N.; Bursal, E.; Aras, A.; Mantarci, A.; Colak, N.; Turkan, F.; Gulcin, I. Synthesis, characterization, powder X-ray diffraction analysis, thermal stability, antioxidant properties and enzyme inhibitions of M (II)-Schiff base ligand complexes. J. Biomol. Struct. Dyn. 2020, 4, 1-8, https://doi.org/10.1080/07391102.2020.1802340.

21. Savir, S.; Wei, Z.J.; Liew, J.W.; Vythilingam, I.; Lim, Y.A.; Saad, H.M.; Sim, K.S.; Tan, K.W. Synthesis, cytotoxicity and antimalarial activities of thiosemicarbazones and their nickel (II) complexes. J. Mol. Struct. 2020, 1211, 128090, https://doi.org/10.1016/j.molstruc.2020.128090.

22. Malekshah, R.E.; Shakeri, F.; Khaleghian, A.; Salehi, M. Developing a biopolymeric chitosan supported Schiff-base and $\mathrm{Cu}$ (II), Ni (II) and Zn (II) complexes and biological evaluation as pro-drug. Int. J. Biol. Macromol. 2020, 152, 846-861, https://doi.org/10.1016/j.ijbiomac.2020.02.245. 
23. Szklarzewicz, J.; Jurowska, A.; Matoga, D.; Kruczała, K.; Kazek, G.; Mordyl, B.; Sapa, J.; Papiez, M. Synthesis, coordination properties and biological activity of vanadium complexes with hydrazone Schiff base ligands. Polyhedron. 2020, 185, 114589, https://doi.org/10.1016/j.poly.2020.114589.

24. Rambabu, A.; Ganji, N.; Daravath, S.; Venkateswarlu, K.; Rangan, K. Mononuclear Co (II), Ni (II) and Cu (II) complexes of the Schiff base, 2-(((4-trifluoromethoxy) phenylimino) methyl)-6-tert-butylphenol: Synthesis, spectroscopic characterization, X-ray study, and biological evaluation. J. Mol. Struct. 2020, 1199, 127006, https://doi.org/10.1016/j.molstruc.2019.127006.

25. Bian, M.; Wang, X.; Sun, Y.; Liu, W. Synthesis and biological evaluation of gold (III) Schiff base complexes for the treatment of hepatocellular carcinoma through attenuating TrxR activity. Eur. J. Med. Chem.2020, 193, 112234, https://doi.org/10.1016/j.ejmech.2020.112234.

26. Santosh Kumar, S.; Priyadarsini, K.I.; Sainis, K.B. Free radical scavenging activity of vanillin and o-vanillin using 1,1-diphenyl-2-picrylhydrazyl (DPPH) radical. Redox. Rep. 2002, 7, 35-40, https://doi.org/10.1179/135100002125000163.

27. Andrews, J.M. BSAC standardized disc susceptibility testing method (version 7). J. Antimicrob. Chemother. 2008, 62, 256-278, https://doi.org/10.1093/jac/dkn194. 